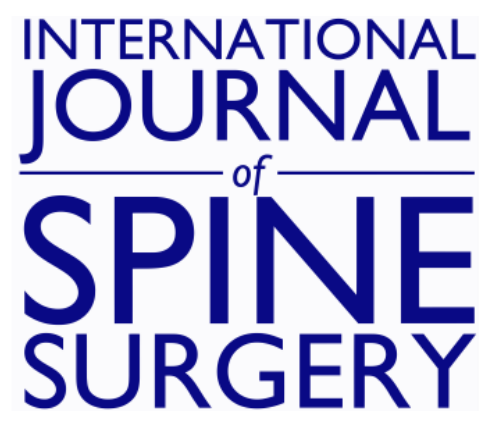

\title{
Robotics and Spine Surgery: Lessons From the Personal Computer and Industrial Revolutions
}

Ahilan Sivaganesan, Nicholas J. Clark, Ram K. Alluri, Avani S. Vaishnav and Sheeraz A. Qureshi

Int J Spine Surg 2021, 15 (s2) S21-S27

doi: https://doi.org/10.14444/8137

http://ijssurgery.com/content/15/s2/S21

This information is current as of April 26, 2023.

Email Alerts Receive free email-alerts when new articles cite this article. Sign up at:

http://ijssurgery.com/alerts

The International Journal of Spine Surgery

2397 Waterbury Circle, Suite 1,

Aurora, IL 60504, Phone: +1-630-375-1432 


\title{
Robotics and Spine Surgery: Lessons From the Personal Computer and Industrial Revolutions
}

\author{
AHILAN SIVAGANESAN, MD,${ }^{1}$ NICHOLAS J. CLARK, MD,${ }^{1}$ RAM K. ALLURI, MD,${ }^{1}$ AVANI S. \\ VAISHNAV, MBBS, ${ }^{1}$ SHEERAZ A. QURESHI, MD $^{1,2}$ \\ ${ }^{1}$ Hospital for Special Surgery, New York, New York, ${ }^{2}$ Weill Cornell Medical College, New York, New York
}

\begin{abstract}
Over the past decade, several review articles have evaluated the techniques and outcomes of robotics vs traditional methods in spine surgery. Recently, robot-assisted pedicle screw placement has emerged, representing an important milestone in the evolution of spine surgery. In the present article, the authors aim to provide the historical context regarding the use and growth of spinal robotics through the lens of the Industrial Revolution and the personal computer revolution. While the former provides insight into the current implications of robotics in spine surgery, the latter predicts future steps in this arena.
\end{abstract}

Special Issue Article

\section{INTRODUCTION}

The advent of robot-assisted pedicle screw placement represents a significant milestone in the evolution of spine surgery. While the adoption of spinal robotics has not yet become widespread, the literature now abounds with studies documenting its feasibility and comparative effectiveness in relation to traditional methods. ${ }^{1-3}$ Multiple review papers summarizing the techniques and outcomes of spinal robotics have also now been published. ${ }^{4-10}$ What does not yet exist, however, is an attempt to frame the arrival of spinal robotics in a historical context that extends beyond the scope of healthcare. Here, we aim to provide exactly this context. Specifically, we draw parallels between spinal robotics and two historical phenomena: the Industrial Revolution (IR) and the personal computer revolution. The breakthroughs and principles of the IR provide a powerful lens through which to understand the current implications of robot-assisted spine surgery. Meanwhile, a careful study of the personal computer revolution provides meaningful lessons and forecasts for the future directions of spinal robotics.

\section{INDUSTRIAL REVOLUTION}

\section{Machine Tools}

Before the Industrial Revolution (IR), most woodworking and metalworking was performed by skilled artisans. These individuals had the ability to fashion a vast array of products; however, the quality was variable, the process was time and labor intensive, and components were rarely interchangeable. ${ }^{11}$ A key catalyst of the IR was the introduction of machine tools, which decreased society's dependence on skilled artisans. Consider a task that requires the application of a cutting tool to a workpiece. Historically, the artisan would "freehand" this task with the goal of achieving an end result that matched the intended specifications. Machine tools simplified this process by constraining the position of the workpiece and the tool such that the movement of one relative to the other is guided. ${ }^{12}$ For example, metal cutting machines create a "toolpath" with the aid of electric power. Early examples of machine tools were the screwcutting lathe, the metal planer, and the milling machine. ${ }^{12}$ Machine tools brought an unprecedented level of accuracy and precision to metalwork, which laid the foundation for interchangeable parts, a major pillar of mass production.

Robot-assisted pedicle screw placement is a direct application of the machine tool concept to spinal instrumentation. The robotic arm defines a specific "toolpath" between the patient's spine (the "workpiece") and the surgeon's tools (such as a drill, tap, and screwdriver). If the surgeon uses those tools within the constraints of the robotic arm, he/she will place screws precisely as planned. The parallel here is striking; the traditional method of pedicle screw 
placement is known as the "freehand" technique, and pre-IR metalworking by artisans was also known as freehand or offhand. It is not merely a matter of semantics, however. Just as machine tools enabled items to be produced consistently with prespecified dimensions, there is now evidence that robot-assisted pedicle screw placement is more accurate than twodimensional fluoroscopy or three-dimensional navigation-based methods. ${ }^{2,3,7-9,13,14}$

\section{Mass Production}

The advent of machine tools was a critical stepping stone toward mass production, which was a hallmark of the IR. Before the IR, most goods were created using "craft production." This consisted of a skilled craftsman gathering and assembling components of an item, retrieving multiple tools necessary for various steps, measuring and then remeasuring after certain steps, and relying on a combination of discipline and "feel" to complete the task successfully. ${ }^{15}$ There were inconsistencies in the quality of goods because of variations in the craftsmen's skill level, the quality of his/her shop, the fatigue factor, and more. With the widespread adoption of machine tools, also known as mechanization, this no longer became the case. With the mechanization of manufacturing, it was said that the "skill is built into the tool" and the "craftsmanship is in the workbench itself." Factories were designed around machine tools that were organized and positioned strategically to minimize unnecessary human motions. This improved the reliability of products and greatly increased productivity.

The spine surgeon who works without robot assistance can be likened to the expert craftsman. Just as the craftsman of old would have to make "cut-and-try" changes to various aspects of a workpiece to complete a task, traditional surgeons often make multiple adjustments when placing pedicle screws freehand or with image guidance. They may need to palpate the bony tract more than once before screw placement, reposition a screw that has caused a cortical breach, or adjust the height of a screw head to facilitate rod placement. Multiple xrays may be taken during this process. By contrast, robot-assisted screw placement can be performed with fewer confirmatory steps and adjustments (improved economy of motion), with the assurance that final hardware placement is consistent with the preoperative plan. Current robotic systems require just three steps, drill, tap, and insert screw, whereas freehand or even image-guided placement can require the following steps: cannulate, feel, measure, tap, feel, and insert screw. While the current generation of robots may not be able to match the best surgeons with respect to the speed of instrumentation, it is likely that the robot will increase the efficiency of surgeons across the spectrum. Just as mechanization enabled mass production in the IR, robot assistance in the operating room may increase surgeons' productivity in the years to come.

\section{Computer-Aided Manufacturing (CAM)}

CAM was initially defined as the use of software to control machine tools. It has since been broadened to include the use of software to aid in the myriad aspects of factory operations: planning, transport, storage, and energy management. ${ }^{16}$ The fundamental technology of CAM is computer numerical control, wherein a machine manipulates raw materials (metal, wood, etc) to match certain specifications by executing preprogrammed software code. ${ }^{17}$ The use of computers has improved the precision and standardization of manufacturing while also transforming the planning process that occurs beforehand. Computer-aided design allows items to be designed down to the last quantitative detail using software, after which those designs are translated into CAM code for actual production. ${ }^{17}$

The advent of robotics in spine surgery is a direct manifestation of CAM technology in the operating room. In fact, the robotic arm that is used by most major surgical vendors is a computer numerical control machine akin to what is found in manufacturing plants. ${ }^{18}$ The promise of increased surgical precision is widely understood; however, there are limits of CAM that are relevant to robot-assisted surgery. One issue is that machines will only produce an item that is as accurate as the programming allows. Similarly, the accuracy of robot-assisted pedicle screw placement is dictated by the quality of imaging registration and preoperative planning. A second issue with CAM is that improper insertion of raw materials can lead to flawed products, even if the programming is perfect. The same is true in surgery; improper loading of the drill, tap, or screwdriver through the robotic arm (with deflection away from the desired trajectory) increases the risk of malpositioned pedicle screws. Furthermore, CAM is limited by the quality of the raw materials themselves, and robot-assisted spinal instrumentation is also limited by the quality of the 
implants. However, one advantage of CAM should not be overlooked: the computer numerical control machine is not subject to the fatigue of a human worker. In the same vein, the robotic arm in the operating room possesses the same level of precision when placing the first screw as it does when placing the last screw in a multilevel instrumentation case. This is often not the case when surgeons place multiple screws in a free-hand manner (or with navigation assistance); muscle fatigue and loss of focus can come into play. For a summary of all of these parallels between the IR and spinal robotics, see Table 1.

\section{PERSONAL COMPUTER REVOLUTION}

\section{Moore's Law}

Before the 1970s, computers were prohibitively expensive and large enough to fill entire rooms. They were only used by universities, government agencies, and large companies, and their primary function was to handle calculations that were difficult to do manually. If multiple individuals wanted to access a central computer, they would take part in time sharing through separate computer terminals. ${ }^{19}$ All of this changed with the advent of the microprocessor. With the microprocessor, an entire central processing unit could fit on a single integrated circuit, and this allowed the cost and size of computers to start decreasing precipitously. ${ }^{20}$ The reason for this is the infamous Moore's Law of 1965 that accurately predicted that the density of transistors, which could be fit onto a microchip, would increase by "a factor of two per year." Of note, this law speaks not only to the achievable transistor density on a chip but also to the transistor density for which the cost per transistor is lowest. ${ }^{20}$ The fulfillment of Moore's Law led to successively smaller, cheaper, and more powerful computers, with a seminal example being the Altair 8800 that was popularized in $1975 .^{21}$ Although the Altair was essentially a hobbyist's item, true personal computers arrived soon after in the form of the "1977 Trinity," the Apple II, the PET 2001, and the TRS$80 .^{21}$ In the $1980 \mathrm{~s}$ and $1990 \mathrm{~s}$, Microsoft rose to prominence as a software company and provided the operating system for modern personal computers.

The state of spinal robotics is perhaps analogous to the state of computing in the 1960s and 1970s. Current robotic solutions are large, often unwieldy, expensive, and only available in select institutions. Some surgeons feel that they only have value for the most complex spinal deformities, just as the original computers were used mainly for particularly challenging calculations. If history is any guide, spinal robotics will become less expensive, less unwieldy, and more powerful with every passing year, even if the pace of innovation does not quite match Moore's Law. After all, the critical technology for a robot is nothing more than hardware and software. One could even surmise that spinal robotics currently has its own version of the "1977 Trinity," the ExcelsiusGPS from Globus, the Mazor X Stealth from Medtronic, and the Rosa One Spine from Zimmer Biomet. Computers in the 1980s and 1990s became ubiquitous and multifunctional, and robots are likely to follow a similar trajectory in the future. There was a time when computers were useful only for laborious calculations and largescale data management, just as robot assistance in the operating room is largely confined to pedicle screw placement. In the coming years, spinal robots will likely be found in most hospitals and may be able to assist not only with instrumentation but also with neural decompression, decortication, deformity correction, and wound closure. An entire industry focused on the software infrastructure for those robots will also surely emerge, just as Microsoft and other companies capitalized on the software demand created by personal computers.

\section{Internet Connectivity}

The modern-day internet would have been difficult to imagine even in the early 1990s, as personal computers were becoming ubiquitous. The roots of the internet date back to the 1960s, when the Pentagon's Advanced Research Projects Agency developed protocols for basic time sharing and message transmissions between computers. This soon evolved into "ARPANET," which connected together an array of academic and military computer networks. ${ }^{22}$ Eventually, commercial networks and personal computers (and now mobile devices) were incorporated, leading to the widespread connectivity we enjoy today. Some manifestations of the internet were perhaps predictable to some degree, such as the World Wide Web, email, and file sharing. Other developments, such as online retail, music and video streaming, social networking, and cloud computing were not easily anticipated. 
Table 1. Parallels between the Industrial Revolution and spinal robotics.

\begin{tabular}{ll}
\hline Industrial Revolution & \multicolumn{1}{c}{ Spinal Robotics } \\
\hline Skilled artisans & Traditional surgeons \\
Machine tools, "toolpaths" & Robot-assisted pedicle screw placement \\
Mechanization, mass production & Improved efficiency with instrumentation \\
Computer-aided design/machining & Preoperative/intraoperative instrumentation planning \\
\hline
\end{tabular}

A critical observation here is that computers, in isolation, did improve the productivity of individuals. However, it was the networking of computers in the form of the internet that unleashed an entirely new set of capabilities. The same principle can be applied to spinal robotics. Currently available robots assist with instrumentation and may one day facilitate other procedural steps, and this will be a boon for surgical productivity and outcomes. However, communication and networking among multiple robots across space and time could open up even more possibilities. Robots represent the first real opportunity for intraoperative data gathering, and the pooling of such data from hundreds or thousands of surgeries would create a robust foundation for machine learning and artificial intelligence (Figure). One can imagine a future scenario where a surgeon is planning instrumentation or decompression maneuvers, and the robot leverages an archive of historically similar patients to make intelligent suggestions that minimize the risk of pseudoarthrosis or durotomy. Another advantage of networking spinal robots would be the opportunity for remote intraoperative consultations. A surgeon who wants a second opinion or "set of eyes" at a decision point during a surgery

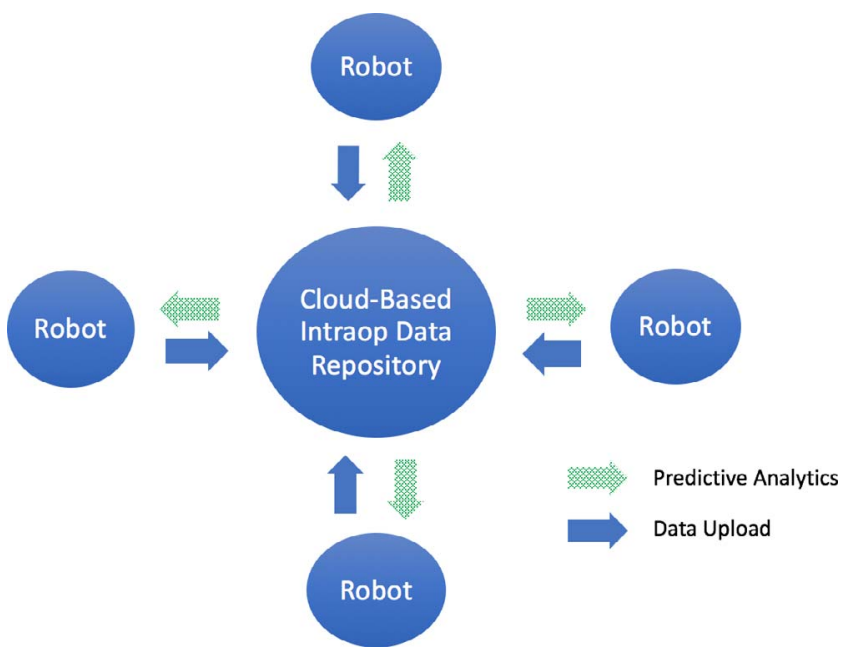

Figure. In the intraoperative data sharing of the future, data from hundreds or thousands of robotic-assisted surgeries can be pooled to enhance machine learning and artificial intelligence. could share the robot's operative data through the cloud with a colleague who uses a compatible system. The colleague could view the information (navigational imaging, spinal parameters, etc) from a computer or mobile device and make recommendations. Interconnectivity among spinal robots could transform them from simple mechanical aids to comprehensive data platforms that allow for the reimagining of clinical trials, prospective registries, and telemedicine.

\section{Software Applications}

It is a common assumption that the personal computer revolution is fundamentally about developments in hardware technology. While advances in microchip technology were instrumental, it has been the advent of specific software applications that has allowed that technology to be relevant to society in general. An early example is Visicalc, which was a spreadsheet application released for the Apple II in $1979 .^{23}$ It was a powerful tool for home- and business-based accounting, and it became known as the Apple II's "killer app." It was even said that Visicalc could be "the software tail that wags (and sells) the personal computer dog" and that computers were being sold as a "Visicalc accessory." Many historians credit Visicalc with transforming computers from a hobbyist's attraction to a general-use device. A much more recent example is the mobile version of Google Maps. This software enabled individuals to take advantage of global positioning system technology through their smartphones, and it led some observers to state that "the paperless map is the killer app." Software such as this, which took full advantage of mobile technology, is credited with catalyzing the rise of the smartphone.

The potential parallels with spinal robotics are self-evident. Robots represent an enabling technology for surgeons, but it will be the development of seminal software applications ("killer apps") that reveal their true potential in the years to come. Even now, preoperative and intraoperative planning of instrumentation, image registration, and spinal segmentation all represent key functionalities that 
Table 2. Parallels between the personal computer revolution and spinal robotics.

\begin{tabular}{ll}
\hline Personal Computer Revolution & \multicolumn{1}{c}{ Spinal Robotics } \\
\hline Moore's Law & Smaller, cheaper, and more powerful robots \\
"1977 Trinity": Apple II, PET 2001, and TRS-80 & "2020 Trinity": ExcelsiusGPS, Mazor X Stealth, and Rosa One Spine \\
Internet connectivity & Intraoperative data sharing \\
"Killer apps": Visicalc and Google Maps & Procedural apps, robotic decompression \\
\hline
\end{tabular}

are made possible by software and stand apart from core robotics technology. In the future, one can imagine software that allows for robot-assisted decompressions, real-time predictions of alignment changes after osteotomies and rod persuasion, and even continuous assessments of surgeon fatigue. The iPhone ushered in the era of the "App Store," wherein third-party developers create applications for an increasing number of users' desires and needs, and a similar phenomenon is likely with robotics. We can envision a situation in the near future where the main robot vendors and outside software developers create applications for specific surgical scenarios. There may be an application specifically for robotic assistance during a transforaminal lumbar interbody fusion, another application for a T10-pelvis posterior fusion, another application for a lateral lumbar interbody fusion, and yet another for a revision decompression. It is quite possible that certain seminal applications will be "the software tail that wags the spinal robotics dog" and that surgeons may finally adopt robotics en masse because they are drawn to particular software features rather than the machines themselves. For a summary of all of these parallels between the personal computer revolution and spinal robotics, see Table 2.

\section{THE FUTURE OF ROBOT-ASSISTED SPINE SURGERY}

While the IR serves as a historical analogy for what robot-assisted spine surgery has currently achieved, the personal computer revolution provides insights into what the future may hold. If research and development for spinal robotics is focused on mirroring key developments in the personal computer revolution, the technologies will advance at an accelerated pace. It is simply a matter of drawing the appropriate lessons from history.

Computers took a leap forward when they became multifunctional as opposed to single-purpose machines dedicated only to large-scale calculations and data storage. ${ }^{21}$ Spinal robots will similarly gain additional traction if and when they are able to assist with portions of surgeries beyond pedicle screw insertion. Examples of potential capabilities include assistance with neural decompression, decortication, deformity correction, and wound closure. Neural decompression is the single most common goal of spine surgery, so if robots could assist with this task, their scope of use would increase dramatically. The critical breakthrough for this capability would be magnetic resonance imaging-based image registration. If a robot's software could process a patient's magnetic resonance imaging data, it would hypothetically be possible to define precise segments of bone or ligament that are to be resected with a robotic arm-assisted burr or Kerrison. Similarly, decortication could be accomplished by using standard computed tomography-based image registration to define specific segments of bone that are to be roughened with a robotic arm-assisted burr. Moreover, deformity corrections could be facilitated by having robotic software calculate the ideal rod contour based on an intraoperative computed tomography "spin" that is performed after instrumentation and osteotomies. And finally, myofascial wound closures could be facilitated by using a stereotactic pointer to identify target points for suture throws. A robotic arm could then theoretically pass sutures through those target points, with the surgeon tying knots at either end of the wound. It should be noted that many of the aforementioned capabilities would be more practical if robotic software could continuously update image registration as spinal anatomy changes during the course of a surgery.

The power of personal computing rose dramatically with the advent of internet connectivity, and spinal robots could follow the same path. This requires that the robots be seen not only as "enabling technologies" for surgeons but also as individual nodes in a network of intraoperative data. A key observation here is that as robotic assistance is applied to ever-increasing portions of spine procedures, the robots will become data repositories of intraoperative maneuvers. For the first time in the history of spine surgery, it will be 
possible to accurately quantify the following variables in real-time: the location and extent of decompressions, the thoroughness of arthrodesis preparation, the positioning of instrumentation, and spinal alignment. When these data from multiple robots and multiple surgeries are combined at scale, the potential for quality improvement and comparative effectiveness research will be boundless. Cloud-based data sharing between robotic workstations will also enable image-based intraoperative consultations among spine surgeons.

A third insight from the personal computer revolution is the primacy of software over hardware. Rather than assuming that research and development for spinal robotics must focus primarily on the machines themselves, innovators in the field should also prioritize the development of software that is based on user (surgeon)-centered design. If "killer apps" are created that dramatically improve surgeons' operative experience, they will be compelled to adopt the technology even if they were initially skeptical of robotics. As has been the case with personal computers, the most certain way to catalyze development of such apps is for robotics companies to bring in third-party developers. If the companies create applicationprogrammable interfaces that allow those thirdparty developers to create software for the robots, an "App Store"-style marketplace will emerge. This will trigger an explosion in the capabilities of spinal robotics.

\section{CONCLUSION}

Robotics has the potential to usher in an IR for spine surgery. The technology incorporates key elements from the historical IR: machine tools, mass production, computer-aided machining, and design. It also stands to reason that robotics will follow the arc of the personal computer revolution in the years to come. The robots will become smaller, less expensive, and more powerful. Interconnectivity among robots will reframe them as a network of intraoperative data repositories, and software applications will expand their capabilities far beyond assistance with instrumentation. Studying historical analogs from other industries can help one understand the underlying value of a new technology as well as maximize its future potential. The goal of this review was to accomplish this for spinal robotics.

\section{REFERENCES}

1. Fayed I, Tai A, Triano M, et al. Robot-assisted percutaneous pedicle screw placement: evaluation of accuracy of the first 100 screws and comparison with cohort of fluoroscopy-guided screws. World Neurosurg. 2020;143:e492e502. doi:10.1016/j.wneu.2020.07.203

2. Mason A, Paulsen R, Babuska JM, et al. The accuracy of pedicle screw placement using intraoperative image guidance systems. J Neurosurg Spine. 2014;20(2):196-203. doi:10.3171/ 2013.11.SPINE13413

3. Shin MH, Hur JW, Ryu KS, Park CK. Prospective comparison study between the fluoroscopy-guided and navigation coupled with O-arm-guided pedicle screw placement in the thoracic and lumbosacral spines. J Spinal Disord Tech. 2015;28(6):E347-E351. doi:10.1097/BSD.0b013e31829047a7

4. Gao S, Lv Z, Fang H. Robot-assisted and conventional freehand pedicle screw placement: a systematic review and meta-analysis of randomized controlled trials. Eur Spine J. 2018;27(4):921-930. doi:10.1007/s00586-017-5333-y

5. Tarawneh AM, Salem KM. A systematic review and meta-analysis of randomized controlled trials comparing the accuracy and clinical outcome of pedicle screw placement using robot-assisted technology and conventional freehand technique. Global Spine J. 2020;11(4):575-586. doi:10.1177/ 2192568220927713

6. Liu H, Chen W, Wang Z, Lin J, Meng B, Yang H. Comparison of the accuracy between robot-assisted and conventional freehand pedicle screw placement: a systematic review and meta-analysis. Int J Comput Assist Radiol Surg. 2016;11(12):2273-2281. doi:10.1007/s11548-016-1448-6

7. Tian NF, Huang QS, Zhou P, et al. Pedicle screw insertion accuracy with different assisted methods: a systematic review and meta-analysis of comparative studies. Eur Spine $J$. 2011;20(6):846-859. doi:10.1007/s00586-010-1577-5

8. Perdomo-Pantoja A, Ishida W, Zygourakis C, et al. Accuracy of current techniques for placement of pedicle screws in the spine: a comprehensive systematic review and metaanalysis of 51,161 screws. World Neurosurg. 2019;126:664-678. doi:10.1016/j.wneu.2019.02.217

9. Meng XT, Guan XF, Zhang HL, He SS. Computer navigation versus fluoroscopy-guided navigation for thoracic pedicle screw placement: a meta-analysis. Neurosurg Rev. 2016;39(3):385-391. doi:10.1007/s10143-015-0679-2

10. Fatima N, Massaad E, Hadzipasic M, Shankar GM, Shin JH. Safety and accuracy of robot-assisted placement of pedicle screws compared to conventional free-hand technique: a systematic review and meta-analysis. Spine J. 2020;21(2):181192. doi:10.1016/j.spinee.2020.09.007

11. Roe JW. English and American Tool Builders. New York, NY: McGraw-Hill; 1926.

12. Moore WR. Foundations of Mechanical Accuracy. Bridgeport, CT: Moore Special Tool Co; 1970.

13. Solomiichuk V, Fleischhammer J, Molliqaj G, et al. Robotic versus fluoroscopy-guided pedicle screw insertion for metastatic spinal disease: a matched-cohort comparison. Neurosurg Focus. 2017;42(5):E13. doi:10.3171/2017.3.FOCUS1710

14. Schatlo B, Molliqaj G, Cuvinciuc V, Kotowski M, Schaller K, Tessitore E. Safety and accuracy of robot-assisted versus fluoroscopy-guided pedicle screw insertion for degenerative diseases of the lumbar spine: a matched cohort compar- 
ison. J Neurosurg Spine. 2014;20(6):636-643. doi:10.3171/2014. 3.SPINE13714

15. Emerson HP, Naehring DCE. Origins of Industrial Engineering: The Early Years of a Profession. Norcross, GA: Industrial Engineering \& Management Press, Institute of Industrial Engineers; 1988.

16. Pichler F, Moreno-Díaz R (eds). Computer Aided Systems Theory, EUROCAST '91: a selection of Papers from the Second International Workshop on Computer Aided Systems Theory, Krems, Austria, April 15-19, 1991: Proceedings. New York, NY: Springer; 1992.

17. Bi Z, Wang X. Computer Aided Design and Manufacturing. Hoboken, NJ: Wiley-ASME Press; 2020.

18. Lanfranco AR, Castellanos AE, Desai JP, Meyers WC. Robotic surgery: a current perspective. Ann Surg. 2004;239(1):14-21. doi:10.1097/01.sla.0000103020.19595.7d

19. Corbató FJ, MIT Computation Center. The Compatible Time-Sharing System: A Programmer's Guide. Cambridge, MA: MIT Press; 1963.

20. Flamm K. Measuring Moore's Law: Evidence from Price, Cost, and Quality Indexes. Cambridge, MA: National Bureau of Economic Research; 2018. NBER working paper series working paper 24553.

21. Ceruzzi PE. A History of Modern Computing. 2nd ed. Cambridge, MA: MIT Press; 2003.

22. Abbate J. Inventing the Internet. Cambridge, MA: MIT Press; 2000.

23. Hill CWL, Jones GR, Schilling MA. Strategic Management: Theory \& Cases: An Integrated Approach. 11th ed. Boston, MA: Cengage Learning; 2014.

Disclosures and COI: Dr Qureshi receives royalties from and provides consulting services for Globus Medical, Inc, and Stryker K2M, has private investments in Tissue Differentiation Intelligence, has speaking and/or teaching arrangements with AMOpportunities (Honoraria), Globus Medical, Inc (Speakers' Bureau), and RTI Surgical Inc, and served on the Scientific Advisory Board for Healthgrades (past relationship), Lifelink.com Inc, and Spinal Simplicity, LLC. Other disclosures include Simplify Medical, Inc (clinical events committee member); Globus Medical, Inc, Integrity Implants Inc, Medical Device Business Services, Medtronic USA, Inc, Nuvasive, Inc, Paradigm Spine, and Stryker K2M (trips/travel); the Society of Minimally Invasive Spine Surgery (Board of Directors); the Annals of Translational Medicine, Contemporary Spine Surgery (Editorial Board Member); the Association of Bone And Joint Surgeons, Cervical Spine Research Society, International Society for the Advancement of Spine Surgery, Lumbar Spine Research Society, North American Spine Society, and Society of Minimally Invasive Spine Surgery (Committee Member); and the Minimally Invasive Spine Study Group and Society of Minimally Invasive Spine Surgery (Board Member). All other authors have nothing to disclose.

Corresponding Author: Ahilan Sivaganesan, MD, Hospital for Special Surgery, 535 E 70th St, New York, NY 10021; Phone: (856) 671-1224; Fax: (215) 955-1113; Email: ahilan.sivaganesan@gmail. com.

Published 26 October 2021

This manuscript is generously published free of charge by ISASS, the International Society for the Advancement of Spine Surgery. Copyright (C) 2021 ISASS. To see more or order reprints or permissions, see http://ijssurgery.com. 